\title{
Sleep quality and associated factors among pregnant women attending antenatal care at Jimma Medical Center, Jimma, Southwest Ethiopia, 2020: cross- sectional study
}

Tamrat Anbesaw ${ }^{1 *}$, Habtamu Abebe ${ }^{2}$, Chalachew Kassaw ${ }^{3}$, Tilahun Bete ${ }^{4}$ and Alemayehu Molla ${ }^{3}$

\begin{abstract}
Background: Sleep is a natural physiological process vital for the physical and mental wellbeing of pregnant women and their fetuses. Even though poor sleep quality is a common problem among pregnant women, it is not studied in developing countries including Ethiopia. Therefore, this study was aimed to assess the poor sleep quality and associated factors among pregnant women attending antenatal care at Jimma medical center, Jimma, Southwest Ethiopia, 2020.

Methods: A cross-sectional study design was conducted among 415 pregnant women at Jimma Medical Center (JMC). The study subjects were selected using a systematic random sampling technique. Pittsburgh Sleep Quality Index (PSQI) was used to assess sleep quality using face-to-face interviews. SPSS version 25 was used to analyze data. Bivariate and multivariable logistic regressions were done to identify factors related to sleep quality. In multivariable logistic regression variables with a $p$-value less than 0.05 was considered significant and, adjusted OR (AOR) with $95 \% \mathrm{Cl}$ was used to present the strength of the association.

Results: The prevalence of poor sleep quality among pregnant women was found to be $30.8 \%(95 \% \mathrm{Cl}(26.5,35.2)$. In multivariable analysis, age $\geq 30$ years old ( $\mathrm{AOR}=1.94 ; 95 \% \mathrm{Cl}: 1.03,3.66)$, Multigravida ( $\mathrm{AOR}=1.90 ; 95 \% \mathrm{Cl}$ : 1.90,3.32), depression ( $\mathrm{AOR}=4.26 ; 95 \% \mathrm{Cl}: 2.54,7.14$ ), stress ( $\mathrm{AOR}=1.85 ; 95 \% \mathrm{Cl}: 1.20,3.02$ ) were variables significantly associated with poor sleep quality.

Conclusion: This study found a high prevalence of poor sleep quality among pregnant women. Older age, gravidity, depression, and stress were associated with poor sleep quality. It is better to have routine sleep pattern screening and teach sleep hygiene practice for pregnant women.
\end{abstract}

Keywords: Sleep quality, Pregnancy, Jimma, Ethiopia

* Correspondence: tamratanbesaw@gmail.com

'Department of Psychiatry, College of Medicine and Health Science, Wollo University, P.O. Box 1145, Dessie, Ethiopia

Full list of author information is available at the end of the article

C C The Author(s). 2021 Open Access This article is licensed under a Creative Commons Attribution 4.0 International License, which permits use, sharing, adaptation, distribution and reproduction in any medium or format, as long as you give appropriate credit to the original author(s) and the source, provide a link to the Creative Commons licence, and indicate if changes were made. The images or other third party material in this article are included in the article's Creative Commons licence, unless indicated otherwise in a credit line to the material. If material is not included in the article's Creative Commons licence and your intended use is not permitted by statutory regulation or exceeds the permitted use, you will need to obtain permission directly from the copyright holder. To view a copy of this licence, visit http://creativecommons.org/licenses/by/4.0/ The Creative Commons Public Domain Dedication waiver (http://creativecommons.org/publicdomain/zero/1.0/) applies to the data made available in this article, unless otherwise stated in a credit line to the data. 


\section{Introduction}

Sleep is a physiological process and vital for the normal physical and mental well-being of an individual [1]. Sleep disorder is one of the most common type's mental illnesses, directly related to the thoughts, feelings, and behaviors of humans. Pregnancy is the term given to a woman while her fetus develops inside her uterus [2]. It causes many physical, social, economic, psychological, and hormonal changes contributing to develop different forms of mental illness for a short and long period [3] . In the first trimester period of pregnancy, night urination, shortness of breath, heartburn, forced body position in bed, breast tenderness, and itching are the physiological changes affecting sleep [4]. Quality of sleep is essential for the physical and mental health of both the mother and fetus. A pregnant mother should get at least eight hours of night sleep [5]. The most common sleep problems observed among pregnant women were resting leg syndrome, sleep apnea, insomnia, nocturnal gastroesophageal reflux, and Sleep-related breathing problems [6]. Nearly, 78\% of pregnant women's had any forms of sleep disorder during their pregnancy time and complained of physical problems such as migraine headaches, gestational diabetes, obesity, cardiovascular conditions (hypertension and heart problems), and poor digestion [7]. A pregnant woman who slept less than six hours has a risk for premature birth, pre-eclampsia, prolonged labor, low progesterone level, abruption placenta, miscarriage, fetal death, and low birth weight $[8,9]$. Almost $70 \%$ of mental health conditions including depression, suicidal ideations, and postpartum psychosis happened during pregnancy are due to subjective and objective sleep pattern problems [10]. The prevalence of poor sleep quality among pregnant women in the USA (53-71\%) [11], China (87\%) [12], and Iran (96.4\%) [13].

The determinants of poor sleep quality among pregnant women were socio-economic status, age, divorce, body mass index, first pregnancy, history of fetal death, history of prolonged labor, zinc and fluorine deficiency, gestational period, and having a history of chronic medical illness (DM, hypertension), lack of awareness about sleep hygiene practice, unwanted pregnancy, lack of social support, and history of mental illness $[2,12,14]$.

The most effective intervention for sleep problems related to pregnancy was basic sleep hygiene practice and progressive muscle relaxation techniques [15]. The use of medication for sleep problems related to pregnancy is the last option and better not used in the first trimester of pregnancy [16]. Despite the above prevalence and impact of sleep on the physical and mental well-being of both a mother and a fetus, there is limited study evidence in African countries including Ethiopia. Therefore, this study aimed to assess the prevalence of poor sleep quality and its correlates among pregnant women attending perinatal service at Jimma Medical Center, Southwest Ethiopia,2020.

\section{Methods and materials \\ Study design and period}

An institutional-based cross-sectional study was conducted in August 1-30/ 2020.

\section{Study area}

The study was conducted at Jimma medical center (JMC) antenatal care units. JMC is located in Jimma town, Oromia regional state, which is found in the southern part of Ethiopia $325 \mathrm{~km}$ far away from Addis Ababa. The center gives service to the catchment population of about 15 million people. There was about 9850 pregnant mother who had a follow-up in a year at antenatal care and on average of a typical month 848 pregnant mother visit antenatal care for follow up.

\section{Source population}

All pregnant women who had ANC follow up at Jimma Medical Center.

\section{Study population}

Pregnant women attending the antenatal care follow up during the study period.

\section{Inclusion and exclusion criteria Inclusion criteria}

Women with a gestational age of 4 weeks and above, and aged 18 and above were included in this study.

\section{Exclusion criteria}

Women who were critically ill and difficult to communicate were excluded from the study.

\section{Sampling procedure and sampling techniques Sample size estimation}

The sample size was estimated by using a single population proportion formula. Sample size with z-value of 1.96 and marginal error of 5\% sample was calculated as:

$$
n=\frac{(\mathrm{Z} \alpha / 2)^{2} \times \mathrm{P}(1-\mathrm{P})}{\mathrm{d}^{2}}
$$

Assumption: $\mathrm{n}=$ initial sample size need for this study $\alpha=$ confidence interval (95\%) $\mathrm{p}=$ proportion of $=50 \%$ $(0.5) \mathrm{d}=$ marginal error of $5 \%,(\mathrm{z} \mathrm{\alpha} / 2)^{2}=1.96$.

$$
n=\frac{1.96^{2} \times(0.5(1-0.5)=384}{(0.05)^{2}}
$$

To calculate the sample size, $50 \%$ of the proportion was used since there is no related study done to identify the prevalence of poor sleep quality in Ethiopia. We 
added a $10 \%$ (39) nonresponse rate; finally, the total sample size was 423 .

\section{Sampling procedure}

Systematic random sampling was used to invite respondents within every two intervals while coming to ANC follow-up. The average monthly number of 848 pregnant women visited the hospital. The sampling interval was determined by dividing the total population who had follow-up during a month of data collection period in OPDs of the JMC ANC unit by the sample size. The selected skip interval was by taking total pregnant women of 848 and sample size 423. Therefore, the sampling fraction was $848 / 423 \approx 2$.

\section{Variables}

Dependent variable

Sleep quality: good / poor

\section{Independent variables}

Socio-demographic factors Age, religion, marital status, ethnicity, educational status, income status, employment, pre-pregnancy BMI, and residence.

Obstetrics and clinical factors Gestational age (1st trimester, 2nd trimester, 3rd trimester), gravidity, parity, unplanned pregnancy, maternal depression, anxiety, known history of mental illness, and comorbidity of medical illness (like asthma, cardiovascular, diabetes, hypertension, etc. ...).

Psychosocial factors and behavioral characteristics Social support, stress, alcohol use, khat use, and smoking cigarettes.

\section{Operational definitions}

Sleep quality Using a global scale PSQI good sleep quality (score $=<5$ ) and poor sleep (score $>5$ ) [17].

Depression A total score of $>13$ points using EPDS, was considered as maternal depression [18].

Anxiety Anxiety was assessed using the anxiety subscale adapted from the Depression, Anxiety, and Stress Scale (DASS-21), participants who scored $\geq 8$ were considered as having anxiety [19].

Past and current medical illness Pregnant women who have known chronic medical illness and their diagnosis confirmed in any health institution either governmental or private that currently had follow-up for any chronic medical illness that was assessed by Yes or No questions.

Current substance use Use of at least anyone of substance in the past three months [20].

Perceived stress A cut-off value of 20 participants with a total PSS score more than or equal to 20 will be defined as stressed, meanwhile those with a score $<20$ were grouped as non-stressed [21] .

Social support Using the Maternity Social Support Scale (MSSS) those pregnant mothers were scored $<18$ low social support, score 18-23 (medium social support), and 24-30 (strong social support) [22].

BMI A person was classified as underweight (BMI < $18.5 \mathrm{~kg} / \mathrm{m}^{2}$ ), normal body weight (BMI $18.5-24.9 \mathrm{~kg} /$ $\mathrm{m}^{2}$ ), overweight (BMI $\left.25-29.9 \mathrm{~kg} / \mathrm{m}^{2}\right)$, or obese $(B M I \geq$ $30 \mathrm{~kg} / \mathrm{m}^{2}$ ) [23].

\section{Data collection method and tools}

A structured interviewer-administered questionnaire was used, which has different subunits, questionnaires to assess socio-demographic factors, Pittsburgh sleeping quality index (PSQI), factors that affect sleep quality including obstetrical factors/clinical factors, psychosocial factors /substance use factors.

The outcome variables were assessed using the Pittsburgh Sleep Quality Index (PSQI). The PSQI has 19 items, which are categorized into seven components: subjective sleep quality, sleep latency, sleep duration, habitual sleep efficiency, sleep disturbances, use of sleeping medication, and daytime dysfunction over the last month. Each component scores ranging from 0 to 3 and then getting a global score with an interval from 0 to 21 . This yields sensitivity and a specificity of 89.6 and $86.5 \%$ respectively [17]. The Cronbach alpha of PSQI in the current study was 0.78 .

The Edinburgh Postnatal Depression Scale (EPDS) was used to assess the symptoms of maternal depression. It consists of 10 items and each question has four possible answers with an interval of $0-3$, and a maximum score out of 30. Similar to the previous study, those who score 13 and above were considered as depressed mood [18]. EPDS is a common tool for screening depressive symptomatology; initially, for use during the postnatal periods, it is also additionally validated for use during the perinatal periods in different countries and settings The EPND is validated among the perinatal population in Ethiopia. The Cronbach alpha of EPDS in the current study was 0.91 .

Adapted anxiety subscale from the Depression, Anxiety, and Stress Scale (DASS - 21) wasused to assess 
anxiety. Each item contributes 0 to 3 points to the sum score, resulting in a totalscore that intervals from 0 to 21 , to considered anxiety, the score was 8 and above [19].

Substance use was assessed by ever use of alcohol, Khat, cigarette, and other particular substance for nonmedical purposes in life. The current use of the substance was assessed for the last three months [20]. The presence of a known chronic medical such as hypertension, diabetes mellitus, or others was assessed by yes/ no response.

The perceived stress scale (PSS) was used to assess stress during pregnancy. The PSS has 10 items of selfreport psychological instruments for measuring the perception of stress. Each item contains 0 to 4 points to the sum score resulting in a total score that intervals from 0 to 40 , a higher score indicating greater perceived stress occurring one month before the interview [21]. Social support was assessed by the Maternity Social Support Scale (MSSS). MSSS has three categories; a score of less than 18 is low social support, 18-23 medium social support, and 24-30 high social support) [22].

\section{Data collection procedure}

Data were collected by five trained BSc psychiatry professionals and one supervisor from the MSc student in psychiatry was also trained on how to supervise data. Each section of the questionnaire was prepared in English and then translated into the Amharic and Afan Oromo, and to ensure its understandability and consistency, then back-translated to English by an independent person. Data collectors and a supervisor have received training for two days duration on the purpose of the study, tools, how to collect data, sampling techniques, and how to handle ethical issues including confidentiality. The pretest was done among 21 (5\%) of the sample size pregnant women in Agaro General Hospital before the main study was done to identify impending problems in the proposed study such as data collection tools and to check the performance of the data collectors. The supervisor and principal investigator were made regular supervision to ensure that all necessary data were appropriately collected. Each day throughout the data collection period, the completed questionnaires were assured for completeness and consistency. The collected data were edited and entered into the computer, then checked twice and processed appropriately.

\section{Data processing and analysis}

The collected data were edited, coded, and entered into the Epi Data version 3.1, and then the data was exported to Statistical Package for Social Science (SPSS) 25.0 version for analysis. The bivariate logistic analysis was performed to select candidate variables. All variables with a $p$-value $<0.25$ in the bivariate analysis were entered into the multivariable logistic regression model. Multivariable logistic regression analysis was employed to control for possible confounding effects and to determine the presence of a statistically significant association between independent variables and dependent variables. Hosmer and Lemeshow goodness was used to check the necessary assumptions. The strength of the association was presented by an adjusted odds ratio of $95 \% \mathrm{CI}$ and $P$ value $<0.05$ was considered as statistically significant. Descriptive statistics results containing frequency, percentages, and summary statistics (mean values and standard deviation) were presented to define the study population about relevant variables.

\section{Result \\ Socio-demographic characteristics of participants}

In this study, a total of 415 participants were assessed, with an overall $98.1 \%$ of response rate. The mean (SD) age of the participants was $25.22( \pm 4.62)$ years. More than half $(51.8 \%)$ of respondents were Muslim religious followers. Almost three fourth of the participants (75.4\%) were married and 251(60.5\%) of them were Oromo in their ethnicity. One-third (32.8\%) of participants have attended college and above and the majority 163(39.3\%) were housewives. Most of the respondents, $320(77.1 \%)$ lived in urban areas. More than three fourth, $326(78.6 \%)$ of the participants had a normal body mass index (BMI). More than one-third of (39.8\%) women reported that their average monthly income is below 1000 Ethiopian birr (Table 1).

\section{Obstetrics related characteristics of the participants}

According to this study, nearly half of the study participants $(47.0 \%)$ were in the third trimester in their gestational age. Almost two-thirds (64.8\%) were multigravida and three hundred eight (74.2\%) of the participants were multipara. Eighty-four participants (20.2\%) had reported a previous history of abortion. More than two-thirds $(68.0 \%)$ of the women had a planned pregnancy (Table 2).

\section{Clinical related factors of the participants}

According to this study finding, more than one-quarter of the participant $(27.5 \%)$ and one hundred fourteen (34.0\%) had comorbid depression and anxiety symptoms respectively. Twenty-one $(5.1 \%)$ of respondents had a history of mental illness. From respondents, 23(5.5\%) women had a comorbid medical illness, from these medical illnesses, HIV/AIDS 4(1\%), asthma 5(1.2\%), diabetes $6(1.4 \%)$, and hypertension $8(1.9 \%)$ were reported (Table 3). 
Table 1 Socio-demographic characteristics of pregnant women attending antenatal care at Jimma medical center, Jimma, Southwest Ethiopia, 2020(N=415)

\begin{tabular}{|c|c|c|c|}
\hline Variables & Categories & Frequency $(n=415)$ & Percent (\%) \\
\hline \multirow[t]{3}{*}{ Age in years } & $18-24$ & 200 & 48.2 \\
\hline & $25-29$ & 128 & 30.8 \\
\hline & $\geq 30$ & 87 & 21.0 \\
\hline \multirow[t]{4}{*}{ Religion } & Muslim & 215 & 51.8 \\
\hline & Orthodox & 113 & 27.2 \\
\hline & Protestant & 81 & 19.5 \\
\hline & Others* & 6 & 1.5 \\
\hline \multirow[t]{2}{*}{ Marital status } & Lack of cohabiting partner & 102 & 24.6 \\
\hline & Married & 313 & 75.4 \\
\hline \multirow[t]{6}{*}{ Ethnicity } & Oromo & 251 & 60.5 \\
\hline & Amhara & 69 & 16.6 \\
\hline & Yeme & 35 & 8.4 \\
\hline & Keffa & 29 & 7.0 \\
\hline & Gurage & 20 & 4.8 \\
\hline & Others** & 11 & 2.7 \\
\hline \multirow[t]{4}{*}{ Education status } & Have no formal education & 32 & 7.7 \\
\hline & Primary & 120 & 28.9 \\
\hline & Secondary & 127 & 30.6 \\
\hline & College and above & 136 & 32.8 \\
\hline \multirow[t]{6}{*}{ Occupational status } & Government employed & 88 & 21.2 \\
\hline & Merchant & 38 & 9.2 \\
\hline & Farming & 14 & 3.4 \\
\hline & Student & 67 & 16.1 \\
\hline & Housewife & 163 & 39.3 \\
\hline & Private employed & 45 & 10.8 \\
\hline \multirow[t]{2}{*}{ Residence } & Urban & 320 & 77.1 \\
\hline & Rural & 95 & 22.9 \\
\hline \multirow[t]{4}{*}{ BMI(pre-pregnancy) } & $<18.5 \mathrm{~kg} / \mathrm{m}^{2}$ & 36 & 8.7 \\
\hline & $18.5-24.99 \mathrm{~kg} / \mathrm{m}^{2}$ & 326 & 78.6 \\
\hline & $25-29.99 \mathrm{~kg} / \mathrm{m}^{2}$ & 40 & 9.6 \\
\hline & $\geq 30 \mathrm{~kg} / \mathrm{m}^{2}$ & 13 & 3.1 \\
\hline \multirow[t]{3}{*}{ Average monthly income (Eth. Birr) } & $\leq 1000$ & 165 & 39.8 \\
\hline & $1001-2000$ & 99 & 23.8 \\
\hline & $\geq 2001$ & 151 & 36.4 \\
\hline
\end{tabular}

Others*:-Adventist \&Catholic, **:- Tigre, Wolyita \& Dawro

\section{Psychosocial and substance-related factors of the participants}

From the total of the respondents, about one-third (34.7) of the respondents had stress during pregnancy. Related to social support, more than half (53.3\%), $141(34.0 \%)$, and $53(12.8 \%)$ of the pregnant women had medium social support, high social support, and poor social support respectively. Regarding the current substance use, $19(4.6 \%), 13(3.10 \%)$, and $3(0.7 \%)$ had used alcohol, chewing khat, and smoking a cigarette within the past three months before data collection time respectively (Table 4).

Prevalence of sleep quality among pregnant women

In the current study, the prevalence of poor sleep quality among pregnant women was 30.8\% (95\% CI (26.5, 35.2). Among the total respondents, 98 (23.6\%) rated their overall sleep quality as bad. Below one-half (42.7\%) of 
Table 2 Description of obstetrics-related factors among pregnant women attending antenatal care at Jimma medical center, Jimma, Southwest Ethiopia, 2020(N=415)

\begin{tabular}{llll}
\hline Variables & Categories & Frequency $(\boldsymbol{n}=\mathbf{4 1 5})$ & Percent $(\mathbf{\%})$ \\
\hline Pregnancy by trimester & First trimester & 112 & 27.0 \\
& Second trimester & 108 & 26.0 \\
& Third trimester & 195 & 47.0 \\
Gravidity & Primigravida & 146 & 35.2 \\
& Multigravida & 269 & 64.8 \\
Parity & Nullipara & 107 & 25.8 \\
& Multipara & 308 & 74.2 \\
Current pregnancy status planned & No & 133 & 32 \\
& Yes & 282 & 68 \\
\hline
\end{tabular}

the respondents had faced 31-60 min sleep latency. More than two-thirds (68.9\%) of the respondents had greater than $7 \mathrm{~h}$ of sleep duration per night. The average bedtime of the respondents was 10:05 p.m. Most of the participants, 377 (90.8\%), had $>85 \%$ sleep efficacy. Almost all, 389 (93.7\%) respondents had never used sleeping medication for their sleep disturbance and 82 (19.8\%) of the participants reported that their sleep quality affects their day-to-day function (Table 5).

\section{Factors associated with sleep quality among pregnant} women

Bivariate and multivariable logistic regression analysis was done to identify factors associated with sleep quality among pregnant women. On the bivariate analysis, age, marital status, educational status, residence, income, gestational age, gravidity, unplanned pregnancy, depression, anxiety, stress, social support, and alcohol use showed a $p$-value of $<0.25$ and became a candidate for multivariable analysis. In multivariable binary logistic regression variables; age, gravidity, depression, and stress were found to be statistically associated with sleep quality at a p-value less than 0.05 .

The odds of poor sleep among respondents with the age of older age $(>=30)$ was 1.94 times higher as compared to young age [18-25] $[\mathrm{AOR}=1.94 ; 95 \%$ CI $(1.03,3.66)]$. Those pregnant women with

Table 3 Description of clinical related factors among pregnant women attending antenatal care at Jimma medical center, Jimma, Southwest, Ethiopia, 2020 $(n=415)$

\begin{tabular}{|c|c|c|c|}
\hline Variables & Categories & Frequency $(n=415)$ & Percent $(\%)$ \\
\hline \multirow[t]{2}{*}{ Comorbid depression } & Yes & 114 & 27.5 \\
\hline & No & 301 & 72.5 \\
\hline \multirow[t]{2}{*}{ Past or current mental illness history } & Yes & 21 & 5.1 \\
\hline & No & 394 & 94.9 \\
\hline \multirow[t]{2}{*}{ Anxiety } & Yes & 141 & 34.0 \\
\hline & No & 274 & 66.0 \\
\hline \multirow[t]{2}{*}{ Chronic medical illness } & Yes & 23 & 5.5 \\
\hline & No & 392 & 94.5 \\
\hline \multirow[t]{2}{*}{ HIV/AIDS } & Yes & 4 & 1.0 \\
\hline & No & 411 & 99.0 \\
\hline \multirow[t]{2}{*}{ Asthma } & Yes & 5 & 1.2 \\
\hline & No & 410 & 98.8 \\
\hline \multirow[t]{2}{*}{ Diabetes } & Yes & 6 & 1.4 \\
\hline & No & 409 & 98.6 \\
\hline \multirow[t]{2}{*}{ Hypertension } & Yes & 8 & 1.9 \\
\hline & No & 407 & 98.1 \\
\hline
\end{tabular}


Table 4 Psychosocial and substance factors among pregnant women attending antenatal care at Jimma medical center, Jimma, Southwest, Ethiopia, 2020 $(n=415)$

\begin{tabular}{llll}
\hline Variables & Categories & Frequency( $\boldsymbol{n = 4 1 5 )}$ & Percent (\%) \\
\hline Stress & Yes & 144 & 34.7 \\
& No & 271 & 65.3 \\
Social support & Low social support & 53 & 12.8 \\
& Medium social support & 221 & 53.2 \\
& High social support & 141 & 34.0 \\
Current alcohol use & Yes & 19 & 4.6 \\
& No & 396 & 95.4 \\
Current Khat use & Yes & 13 & 3.1 \\
& No & 402 & 96.9 \\
& No & 3 & 0.7 \\
\hline
\end{tabular}

Table 5 Characteristics of Sleep disturbance among pregnant women attending antenatal care at Jimma medical center, Jimma, Southwest, Ethiopia, 2020 $(n=415)$

\begin{tabular}{|c|c|c|c|}
\hline Variables & Category & Frequency $(\mathbf{n})$ & Percent (\%) \\
\hline \multirow[t]{4}{*}{ Subjective sleep quality } & Very good (0) & 180 & $43.4 \%$ \\
\hline & Fairly good (1) & 137 & $33.0 \%$ \\
\hline & Fairly bad (2) & 82 & $19.8 \%$ \\
\hline & Very bad (3) & 16 & $3.8 \%$ \\
\hline \multirow[t]{4}{*}{ Sleep latency } & $0-15 \min (0)$ & 60 & $14.5 \%$ \\
\hline & $16-30 \min (1)$ & 108 & $26.0 \%$ \\
\hline & $31-60 \min (2)$ & 177 & $42.7 \%$ \\
\hline & $>60 \min (3)$ & 70 & $16.8 \%$ \\
\hline \multirow[t]{4}{*}{ Sleep duration } & $>7 \mathrm{~h}$ & 286 & 68.9 \\
\hline & $6-7 \mathrm{~h}$ & 64 & 15.4 \\
\hline & $5-6 h$ & 43 & 10.4 \\
\hline & $<5 \mathrm{~h}$ & 22 & 5.3 \\
\hline \multirow[t]{4}{*}{ Habitual sleep efficiency } & $\geq 85 \%$ & 377 & 90.8 \\
\hline & $75-84 \%$ & 35 & 8.5 \\
\hline & $65-74 \%$ & 3 & 0.7 \\
\hline & $<65 \%$ & 0 & $0 \%$ \\
\hline \multirow[t]{4}{*}{ Sleep disturbances } & Never (0) & 63 & $15.2 \%$ \\
\hline & 1 time a week (1) & 306 & $73.7 \%$ \\
\hline & 1-2 times a week (2) & 41 & $9.9 \%$ \\
\hline & $\geq 3$ times a week (3) & 5 & $1.2 \%$ \\
\hline \multirow[t]{4}{*}{ Use of sleeping medications } & Never & 389 & $93.7 \%$ \\
\hline & Less than Once a week & 15 & $3.6 \%$ \\
\hline & Once or twice a week & 9 & $2.2 \%$ \\
\hline & Three or more times a week & 2 & $0.5 \%$ \\
\hline \multirow[t]{4}{*}{ Daytime dysfunction over the last month } & No problem (0) & 333 & $80.2 \%$ \\
\hline & $1-2$ times a week (1) & 62 & $14.9 \%$ \\
\hline & 3 times a week (2) & 15 & $3.6 \%$ \\
\hline & $>3$ times a week (3) & 5 & $1.3 \%$ \\
\hline \multirow[t]{2}{*}{ Overall sleep quality } & Good sleep quality & 287 & $69.2 \%$ \\
\hline & Poor sleep quality & 128 & $30.8 \%$ \\
\hline
\end{tabular}


multigravidas were 1.90 times more likely to have poor sleep quality as compared with participants with primigravidas $[\mathrm{AOR}=1.90 ; 95 \% \mathrm{CI} \quad(1.90,3.32)]$. Mothers with comorbid depression were about 4.26 times more likely to have poor sleep quality than their referent groups $[\mathrm{AOR}=4.26 ; 95 \% \mathrm{CI}(2.54,7.14)]$. Likewise, the odds of poor sleep quality among women who had stress was about 1.85 times higher as compared with their counterparts $[\mathrm{AOR}=1.85$; 95\% CI (1.20,3.02)] (Table 6).

\section{Discussion}

The finding of this study showed that the prevalence of poor sleep quality among pregnant women was $30.8 \%$ [(95\% CI, 26.5, 35.2)]. The study finding was higher as compared with a study done in Finland 15\% [24], Peru 17\% [25], \{Venugopal, 2018 \#34\} and China 15.2\% [26]. The variation might be due to the eligibility criteria and sample size. In Peru, pregnant women were between 24 and 28 weeks of pregnancy with 1298 participants while the current study was done on pregnant women

Table 6 Bivariate and multivariate logistic regression analysis results of sleep quality among pregnant women attending ANC at JMC, Jimma, Southwest Ethiopia, 2020 ( N=415)

\begin{tabular}{|c|c|c|c|c|c|c|}
\hline \multirow[t]{2}{*}{ Variables } & \multirow[t]{2}{*}{ Category } & \multicolumn{2}{|l|}{ Sleep quality } & \multirow{2}{*}{$\begin{array}{l}\text { COR } \\
(95 \% \text { C.I) }\end{array}$} & \multirow{2}{*}{$\begin{array}{l}\text { AOR } \\
(95 \% \text { C.I) }\end{array}$} & \multirow[t]{2}{*}{$P$-values } \\
\hline & & Poor sleep quality (n) & Good sleep quality (n) & & & \\
\hline \multirow[t]{3}{*}{ Age } & $18-24$ & $59(29.5 \%)$ & $141(70.5 \%)$ & 1 & 1 & \\
\hline & $25-29$ & $30(23.4 \%)$ & $98(76.6 \%)$ & $0.73(0.44,1.22)$ & $0.98(0.53,1.81)$ & 0.95 \\
\hline & $\geq 30$ & $39(23.4 \%)$ & $48(76.6 \%)$ & $1.94(1.15,3.27)$ & $1.94(1.03,3.66)$ & $0.041^{*}$ \\
\hline \multirow[t]{2}{*}{ Marital status } & Single & $46(45.1 \%)$ & $56(54.9 \%)$ & $2.31(1.45,3.68)$ & $1.47(0.85,2.52)$ & 0.17 \\
\hline & Married & $82(26.2 \%)$ & $231(73.8 \%)$ & 1 & 1 & \\
\hline \multirow[t]{4}{*}{ Educational status } & No formal education & $12(37.5 \%)$ & $20(62.5 \%)$ & $1.66(0.74,3.75)$ & $0.87(0.31,2.40)$ & 0.78 \\
\hline & Primary & $39(32.5 \%)$ & $81(67.5 \%)$ & $1.34(0.78,2.29)$ & $1.46(0.85,2.52)$ & 0.16 \\
\hline & Secondary & $41(32.3 \%)$ & $86(67.7 \%)$ & $1.32(0.78,2.25)$ & $1.01(0.53,1.91$ & 0.99 \\
\hline & College and above & $36(26.5 \%)$ & $100(73.5 \%)$ & 1 & 1 & \\
\hline \multirow[t]{2}{*}{ Residence } & Rural & $35(36.8 \%)$ & $60(63.2 \%)$ & $1.42(0.88,2.31)$ & $0.97(0.54,1.73)$ & 0.92 \\
\hline & Urban & $93(29.1 \%)$ & $227(70.9 \%)$ & 1 & 1 & \\
\hline \multirow[t]{3}{*}{ Income (Ethio birr) } & $\leq 1000$ & $68(41.2 \%)$ & $97(58.8 \%)$ & $2.08(1.29,3.37)$ & $1.71(0.98,2.97)$ & 0.06 \\
\hline & $1001-2000$ & $22(22.2 \%)$ & $77(77.8 \%)$ & $0.85(0.46,1.55)$ & $0.88(0.46,1.71)$ & 0.71 \\
\hline & $\geq 2001$ & $38(25.2 \%)$ & $113(74.8 \%)$ & 1 & 1 & \\
\hline \multirow[t]{2}{*}{ Current pregnancy planned } & No & $46(34.6 \%)$ & $87(65.4 \%)$ & $1.29(0.83,2.01)$ & $0.97(0.57,1.64)$ & 0.91 \\
\hline & Yes & $82(29.1 \%)$ & $200(70.9 \%)$ & 1 & 1 & \\
\hline \multirow[t]{3}{*}{ Gestational age } & First trimester & $45(40.2 \%)$ & $67(59.8 \%)$ & $1.75(1.07,2.86)$ & $1.01(0.56,1.81)$ & 0.99 \\
\hline & Second trimester & $29(26.9 \%)$ & $79(73.1 \%)$ & $0.96(0.56,1.63)$ & $0.72(0.39,1.31)$ & 0.28 \\
\hline & Third trimester & $54(27.7 \%)$ & $141(72.3 \%)$ & 1 & 1 & 0.99 \\
\hline \multirow[t]{2}{*}{ Gravidity } & Primigravida & $31(21.2 \%)$ & $115(78.8 \%)$ & 1 & 1 & \\
\hline & Multigravida & $97(36.1 \%)$ & $172(63.9 \%)$ & $2.09(1.31,3.34)$ & $1.90(1.09,3.32)$ & $0.025^{*}$ \\
\hline \multirow[t]{2}{*}{ Depression } & Yes & $66(57.9 \%)$ & $48(42.1 \%)$ & $5.30(3.33,8.44)$ & $4.26(2.54,7.14)$ & $<0.001^{*}$ \\
\hline & No & $62(20.6 \%)$ & 239(79.4\%) & 1 & 1 & \\
\hline \multirow[t]{2}{*}{ Anxiety } & Yes & $61(43.3 \%)$ & $80(56.7 \%)$ & $2.35(1.53,3.63)$ & $1.10(0.61,1.83)$ & 0.80 \\
\hline & No & $67(24.5 \%)$ & $207(75.5 \%)$ & 1 & 1 & \\
\hline \multirow[t]{2}{*}{ Stress } & Yes & $65(45.1 \%)$ & $79(54.9 \%)$ & $2.72(1.76,4.19)$ & $1.85(1.20,3.02)$ & $0.013^{*}$ \\
\hline & No & $63(23.2 \%)$ & $208(76.8 \%)$ & 1 & 1 & \\
\hline \multirow[t]{3}{*}{ Social support } & Low social support & $26(49.1 \%)$ & $27(50.9 \%)$ & $3.15(1.6,6.13)$ & $1.32(0.60,2.87)$ & 0.49 \\
\hline & Medium social support & $69(31.2 \%)$ & $152(68.8 \%)$ & $1.48(0.92,2.41)$ & $0.99(0.57,1.71)$ & 0.97 \\
\hline & High social support & $33(23.4 \%)$ & 108(76.6\%) & 1 & 1 & \\
\hline \multirow[t]{2}{*}{ Alcohol } & Yes & $13(68.4 \%)$ & $6(31.6 \%)$ & $5.29(1.96,14.26)$ & $2.55(0.85,7.70)$ & 0.10 \\
\hline & No & $115(29.0 \%)$ & $281(71.0 \%)$ & 1 & 1 & \\
\hline
\end{tabular}

*Statistically significant at $P$-value $<0.05$, AOR, Adjusted odds Ratio, $1=$ reference category, Chi square $=8$, Hosmer Lemeshow goodness-of-fit 0.52 , degrees of freedom $=8$, Maximum VIF $=1.42$ 
including all trimesters of gestation, and the sample size was 415 [25]. Another discrepancy might be due to the difference in social support practice, sampling technique, the socio-cultural and demographic context of the women.

However, the current study was lower than the meta-analysis study conducted by Sedov et al. $45.7 \%$ [27], Turkish 86\% [28], Vietnam 41.2\% [29], Iran 77\% [30], Taiwan 60\% [31], and meta-analysis study in China $54.2 \%$ [32]. This discrepancy might be due to variation in used inclusion criteria, sampling technique, study design, and sampling size. A study conducted in Iran included third-trimester pregnancy only, and a convenience sampling technique was used while the current study used a systematic random sampling technique [30]. Another possible reason might be the difference in study design, socioeconomic, sociocultural, and demographic characteristics in the populations.

Regarding factors associated with poor sleep quality, in the current study; older maternal age was nearly two times more likely to have poor sleep during the pregnancy period as compared to younger ones. Sleep quality among pregnant women decreases as the age of the mother increases, this finding was supported by other studies done in Taskiran (Turkish) $(28,29$ and 45-yearold had worse sleep quality than the age group between 17 and 28), Taiwan [33]. China [12], Vietnam [29], and Meta-analysis [27]. The possible reason might be due to usually older mothers have a tendency to have care burdens, heavier domestic responsibilities, more likely to experience physical distresses, and slower recovery from delivery, this causes a probable reason for the decline of sleep quality [34].

This finding also revealed that pregnant women with multigravidas were nearly two times more likely to have poor sleep quality than those who are primigravida. We found that poor sleep quality during pregnancy was associated with multigravidas in cross-sectional analyses. Nevertheless, our findings were inconsistent with those finding from Francisco [35] which only found an association between sleep quality and primigravida. A possible explanation might be multigravidas pregnant mothers complained that their sleep pattern is influenced by their children's sleep habits. When children frequently wake up and/or cry at night, mothers are expected so they also wake up from their sleep to take care of theirs children.

The odds of having poor sleep quality among women who had depression were about 4.26 times higher when compared with the counterpart. This was consistent with the conducted in China [36]. This could be due to the presence of prenatal depression is one of the most possible psychological factors contributing to sleep disturbance during pregnancy [12].

The likelihood of developing poor sleep quality among pregnant women who have stress was 1.85 times higher as compared with no stress. A similar study was done in China [36]. The possible reason might be due to the direct effect of stress during pregnancy on sleep quality might be related to arginine vasopressin hormone, which is involved in the stress response and circadian regulation of the sleep-wake cycle [37].

\section{Conclusion}

Our study found that the prevalence of poor sleep quality among pregnant women was high as compared with studies done in the general population and many other countries. Older age, multigravidas, depression, and stress were statistically significantly associated with poor sleep quality in this study. It is a good alarm to be alert to give attention to routine screening of sleep patterns in pregnant women and to give special concern for pregnant women with the above-stated factors.

\section{Abbreviations \\ AIDS: Acquired Immune Deficiency Syndrome; ANC: Antenatal Care; AOR: Odds Ratio; BMI: Body Mass Index; Cl: Confidence Interval; COR: Crude odds ratio; EPDS: Edinburgh Postnatal Depression Scale; Epi- \\ Data: Epidemiological Data; JMC: Jimma Medical Center; MDD: Major Depressive Disorder; OPD: Out Patient Department; PSQI: Pittsburgh Sleep Quality Index; SD: Standard Deviation; SPSS: Statistical Package for Social Science; USA: United States America}

\section{Acknowledgments \\ The authors would like to thank all the participants of the study.}

Authors' contributions

All authors participated in preparing and approving the manuscript.

Funding

No financial support was received for the study.

Availability of data and materials

The datasets used and/or analyzed during the current study are available from the corresponding author on reasonable request.

\section{Declarations}

Ethics approval and consent to participate

The study was approved by and carried out in accordance with the institutional review board of Jimma University. Informed consent was obtained from all the participants. Written Informed consent was taken from each of the pregnant women and the information from individual mothers was kept confidential, their identity was not shown and there was no dissemination of the information without the respondent's permission. We prepared a private room for an interview; those women who reported sleep disturbance and depression were immediately linked to mental health for further evaluation and management. Interviewers were trained to link participants found to be in physically risky conditions and/or in immediate need of counseling to psychologists and psychiatrists.

Consent for publication

Not applicable.

Competing interests

The authors declare that they have no competing interests. 


\section{Author details}

'Department of Psychiatry, College of Medicine and Health Science, Wollo University, P.O. Box 1145, Dessie, Ethiopia. ${ }^{2}$ Department of Epidemiology and Biostatistics, Faculty of Medical science, Institute of Health Sciences, Jimma University, Jimma, Ethiopia. ${ }^{3}$ Department of Psychiatry, College of Medicine and Health Science, Dilla University, P.O. Box 419, Dilla, Ethiopia. ${ }^{4}$ Department of Psychiatry, College of Medicine and Health Science, Haramaya University, P.O. Box 235, Harar, Ethiopia.

Received: 12 April 2021 Accepted: 7 September 2021

Published online: 26 September 2021

\section{References}

1. Benjamins JS, Migliorati F, Dekker K, Wassing R, Moens S, Blanken TF, et al. Insomnia heterogeneity: characteristics to consider for data-driven multivariate subtyping. Sleep Med Rev. 2017;36:71-81. https://doi.org/10.101 6/.smrv.2016.10.005.

2. Sezgin AU, Punamäki R-L. Impacts of early marriage and adolescent pregnancy on mental and somatic health: the role of partner violence. Arch Womens Ment Health. 2020;23(2):155-66. https://doi.org/10.1007/s00737-01 9-00960-w.

3. Cattane N, Räikkönen K, Anniverno R, Mencacci C, Riva MA, Pariante CM, et al. Depression, obesity and their comorbidity during pregnancy: effects on the offspring's mental and physical health. Mol Psychiatry. 2021;26(2): 462-81. https://doi.org/10.1038/s41380-020-0813-6.

4. Li L, Zhao K, Hua J, Li S. Association between sleep-disordered breathing during pregnancy and maternal and fetal outcomes: an updated systematic review and meta-analysis. Front Neurol. 2018;9:91. https://doi.org/10.3389/ fneur.2018.00091.

5. Facco FL, Parker CB, Reddy UM, Silver RM, Koch MA, Louis JM, et al. Association between sleep-disordered breathing and hypertensive disorders of pregnancy and gestational diabetes mellitus. Obstet Gynecol. 2017;129(1): 31-41. https://doi.org/10.1097/AOG.0000000000001805.

6. Louis JM, Koch MA, Reddy UM, Silver RM, Parker CB, Facco FL, et al. Predictors of sleep-disordered breathing in pregnancy. Am J Obstet Gynecol. 2018;218(5):521. e1-e12.

7. Laposky AD, Pemberton VL. Sleep-disordered breathing and pregnancyrelated cardiovascular disease. J Women's Health. 2021;30(2):194-8. https:// doi.org/10.1089/jwh.2020.8869

8. Wołyńczyk-Gmaj D, Różańska-Walędziak A, Ziemka S, Ufnal M, Brzezicka A, Gmaj B, et al. Insomnia in pregnancy is associated with depressive symptoms and eating at night. J Clin Sleep Med. 2017;13(10):1171-6. https://doi.org/10.5664/jcsm.6764.

9. Liset R, Grønli J, Henriksen RE, Henriksen TE, Nilsen RM, Pallesen S. Sleep, evening light exposure and perceived stress in healthy nulliparous women in the third trimester of pregnancy. PLoS One. 2021;16(6):e252285. https:// doi.org/10.1371/journal.pone.0252285.

10. Moradi S, Zobeiri M, Feizi A, Clark CC, Entezari MH. The Effects of Spirulina (Arthrospira platensis) Supplementation on Anthropometric Indices, Blood Pressure, Sleep Quality, Mental Health, Fatique Status, and Quality of Life in Patients with Ulcerative Colitis: A Randomized, Double-blinded, Placebocontrolled Trial. Int J Clin Pract. 2021;75(10):e14472.

11. Christian LM, Carroll JE, Porter K, Hall MH. Sleep quality across pregnancy and postpartum: effects of parity and race. Sleep Health. 2019;5(4):327-34. https://doi.org/10.1016/.sleh.2019.03.005.

12. Yang Y, Mao J, Ye Z, Zeng X, Zhao H, Liu Y, et al. Determinants of sleep quality among pregnant women in China: a cross-sectional survey. J Matern Fetal Neonatal Med. 2018;31(22):2980-5. https://doi.org/10.1080/14767058.2 017.1359831.

13. Saadati F, Sehhatiei Shafaei F, Mirghafourvand M. Sleep quality and its relationship with quality of life among high-risk pregnant women (gestational diabetes and hypertension). J Matern Fetal Neonatal Med. 2018; 31(2):150-7. https://doi.org/10.1080/14767058.2016.1277704.

14. Yu Y, Zhu X, Xu H, Hu Z, Zhou W, Zheng B, et al. Prevalence of depression symptoms and its influencing factors among pregnant women in late pregnancy in urban areas of Hengyang City, Hunan Province, China: a crosssectional study. BMJ Open. 2020;10(9):e038511. https://doi.org/10.1136/ bmjopen-2020-038511.

15. Briguglio M, Vitale JA, Galentino R, Banfi G, Dina CZ, Bona A, et al. Healthy eating, physical activity, and sleep hygiene (HEPAS) as the winning triad for sustaining physical and mental health in patients at risk for or with neuropsychiatric disorders: considerations for clinical practice. Neuropsychiatr Dis Treat. 2020;16:55-70. https://doi.org/10.2147/NDT.S229206.

16. Amezcua-Prieto C, Naveiro-Fuentes M, Arco-Jiménez N, Olmedo-Requena $R$, Barrios-Rodríguez R, Vico-Zúñiga I, et al. Walking in pregnancy and prevention of insomnia in third trimester using pedometers: study protocol of Walking_Preg project (WPP). A randomized controlled trial. BMC Pregnancy Childbirth. 2020;20(1):1-9.

17. Salahuddin M, Maru TT, Kumalo A, Pandi-Perumal SR, Bahammam AS, Manzar MD. Validation of the Pittsburgh sleep quality index in community dwelling Ethiopian adults. Health Qual Life Outcomes. 2017;15(1):1-7. https://doi.org/10.1186/s12955-017-0637-5.

18. Shoaee F, Mohsenpour Z, Najarzadegan MR, Nekouhi S, Razmjouei P, Babakhanian M, et al. Sensitivity and specificity of the Edinburgh postnatal depression scale (EPDS) among Iranian mothers: a psychometric study. Int J Pediatr. 2019;7(12):10533-40.

19. Lee E-H, Moon SH, Cho MS, Park ES, Kim SY, Han JS, et al. The 21-item and 12-item versions of the depression anxiety stress scales: psychometric evaluation in a Korean population. Asian Nurs Res. 2019;13(1):30-7. https:// doi.org/10.1016/j.anr.2018.11.006.

20. McRee B, Babor TF, Lynch ML, Vendetti JA. Reliability and validity of a twoquestion version of the World Health Organization's alcohol, smoking and substance involvement screening test: the ASSIST-FC. J Stud Alcohol Drugs. 2018;79(4):649-57. https://doi.org/10.15288/jsad.2018.79.649.

21. Manzar MD, Salahuddin M, Peter S, Alghadir A, Anwer S, Bahammam AS, et al. Psychometric properties of the perceived stress scale in Ethiopian university students. BMC Public Health. 2019;19(1):1-8. https://doi.org/10.11 86/s12889-018-6310-z.

22. Demiröz HP, Taştan K. The effects of perceived social support on postpartum depression. J Surg Med. 2018;2(3):298-302.

23. Chandrasekaran A. Body mass index-is it reliable indicator of obesity. J Nutr Weight Loss. 2018;3(1):2. https://doi.org/10.35248/2593-9793.18.3.111.

24. Kataja E-L, Karlsson L, Huizink A, Tolvanen M, Parsons C, Nolvi S, et al. Pregnancy-related anxiety and depressive symptoms are associated with visuospatial working memory errors during pregnancy. J Affect Disord. 2017; 218:66-74. https://doi.org/10.1016/j.jad.2017.04.033.

25. Gelaye B, Addae G, Neway B, Larrabure-Torrealva GT, Qiu C, Stoner L, et al. Poor sleep quality, antepartum depression and suicidal ideation among pregnant women. J Affect Disord. 2017;209:195-200. https://doi.org/10.101 6/j.jad.2016.11.020.

26. Xu X, Liu D, Zhang Z, Sharma M, Zhao Y. Sleep duration and quality in pregnant women: a cross-sectional survey in China. Int J Environ Res Public Health. 2017;14(7):817. https://doi.org/10.3390/ijerph14070817.

27. Sedov ID, Cameron EE, Madigan S, Tomfohr-Madsen LM. Sleep quality during pregnancy: a meta-analysis. Sleep Med Rev. 2018;38:168-76. https:// doi.org/10.1016/j.smrv.2017.06.005.

28. Taskiran N. Pregnancy and sleep quality. J Turkish Soc Obstet Gynecol. 2011; 8(3):181-7. https://doi.org/10.5505/tjod.2011.14880.

29. Huong NTT, Thuy NTH. Quality of sleep among pregnant women. Int J Clin Med. 2018;10(1):16-25.

30. Ahmadi Z, Bakouei F, Bakhtiari A. Maternal sleep quality in late pregnancy: the association between preterm birth and sleep quality. Caspian J Reprod Med. 2019;5(1):17-22.

31. Ko SH, Chang SC, Chen CH. A comparative study of sleep quality between pregnant and nonpregnant Taiwanese women. J Nurs Scholarsh. 2010;42(1): 23-30. https://doi.org/10.1111/j.1547-5069.2009.01326.x.

32. Yang Y, Li W, Ma T-J, Zhang L, Hall BJ, Ungvari GS, et al. Prevalence of poor sleep quality in perinatal and postnatal women: a comprehensive metaanalysis of observational studies. Front Psychiatr. 2020;11:161. https://doi. org/10.3389/fpsyt.2020.00161.

33. Tsai SY, Lee CN, Wu WW, Landis CA. Sleep hygiene and sleep quality of third-trimester pregnant women. Res Nurs Health. 2016;39(1):57-65. https:// doi.org/10.1002/nur.21705.

34. Kamysheva $\mathrm{E}$, Skouteris $\mathrm{H}$, Wertheim EH, Paxton SJ, Milgrom J. A prospective investigation of the relationships among sleep quality, physical symptoms, and depressive symptoms during pregnancy. J Affect Disord. 2010;123(1-3): 317-20. https://doi.org/10.1016/j.jad.2009.09.015.

35. Waters MA, Lee KA. Differences between primigravidae and multigravidae mothers in sleep disturbances, fatigue, and functional status. J NurseMidwifery. 1996;41(5):364-7. https://doi.org/10.1016/50091-2182(96)00049-3.

36. Gao M, Hu J, Yang L, Ding N, Wei X, Li L, et al. Association of sleep quality during pregnancy with stress and depression: a prospective birth cohort 
study in China. BMC Pregnancy Childbirth. 2019;19(1):1-8. https://doi.org/1 0.1186/s12884-019-2583-1.

37. Solomonova E, Lee Y, Robins S, King L, Feeley N, Gold I, et al. Sleep quality is associated with vasopressin methylation in pregnant and postpartum women with a history of psychosocial stress. Psychoneuroendocrinology. 2019;107:160-8. https://doi.org/10.1016/j.psyneuen.2019.05.010.

\section{Publisher's Note}

Springer Nature remains neutral with regard to jurisdictional claims in published maps and institutional affiliations.

Ready to submit your research? Choose BMC and benefit from:

- fast, convenient online submission

- thorough peer review by experienced researchers in your field

- rapid publication on acceptance

- support for research data, including large and complex data types

- gold Open Access which fosters wider collaboration and increased citations

- maximum visibility for your research: over $100 \mathrm{M}$ website views per year

At $\mathrm{BMC}$, research is always in progress.

Learn more biomedcentral.com/submissions 